\title{
DIRECT IMAGE RETRIEVAL IN JPEG AND JPEG2000
}

\author{
K.M. Au, N.F. Law and W.C. Siu \\ Centre for Multimedia Signal Processing \\ Department of Electronic and Information Engineering \\ The Hong Kong Polytechnic University, Hong Kong.
}

\begin{abstract}
Images are often compressed using JPEG or JPEG2000. Many retrieval systems operated in either uncompressed or compressed domains have been proposed. However, retrieving in multiple domains typically involves full decompression for feature analysis in spatial domain. Common features in different domains are thus worth of investigation for direct image indexing. By employing a common subband filtering model, outputs from JPEG and JPEG2000 can be compared directly without having a full decompression. Despite of high compression, similar translation and rotation invariant features can be extracted from these two domains. Simulation results reveal that JPEG and JPEG2000 compressed images can be searched from one another irrespective of the compression ratio. Our experimental studies confirm that retrieval in multiple domains is possible without a full decompression.
\end{abstract}

\section{INTRODUCTION}

With a tremendous increase in the amount of visual data, image indexing and retrieval techniques have become important. These techniques should extract meaningful information (features) from images so that they can be retrieved efficiently based on their contents. In the past decade, retrieval systems for uncompressed spatial domain, such as QBIC [1], have been proposed.

Image/video compression standards such as JPEG and MPEG have been used to reduce storage requirements. Hence, visual data are expected to be stored in compressed form. Most of the compression systems are based on the Discrete Cosine Transform (DCT) or the Wavelet Transform (WT). These different compression techniques result in different transformed coefficients, which preserve different visual characteristics. Currently, the compressed domain-based indexing method greatly depends on their compression techniques. DCT-domain based indexing techniques extract features from the DCT coefficients [2]. Whereas, wavelet domain-based retrieval systems regard significant wavelet magnitudes as features [3].

Due to the various compression standards, images would be available in heterogeneous form. If features could be obtained directly from its compressed form, unnecessary decompression can be avoided. The objective of this paper is to explore common features in different transformed domains so as to facilitate direct image retrieval in their respective domains without a full decompression. Simulation results of image retrieval in joint JPEG and JPEG2000 domains are given.

\section{SUBBAND FILTERING MODEL}

JPEG and JPEG2000 employ dissimilar Block-based Discrete Cosine Transform (BDCT) and Wavelet Transform (WT) as their transformation respectively. Thus, their compressed outputs seem to be irrelevant and cannot be compared directly. Referring to our derived subband filtering model in [4], their spatial-frequency outputs can always be obtained by filtering and a downsampling operation afterwards.

\subsection{Block-based Discrete Cosine Transform in JPEG}

Concatenating BDCT coefficients from all $8 \times 8$ blocks with the same value for $u$, the BDCT output $\tilde{X}_{u, D C T}(z)$ can be obtained by

$$
\tilde{X}_{u, D C T}(z)=\tilde{F}_{u, D C T}(z) \tilde{X}(z) \downarrow 8
$$

where $C(u)$ equals to $1 / \sqrt{2}$ for $u=0$ and 1 otherwise, and

$$
\tilde{F}_{u, D C T}(z)=\frac{C(u)}{2} \sum_{i=0}^{7} \cos \frac{(2 i+1) u \pi}{16} z^{i}
$$

\subsection{Wavelet Transform in JPEG2000}

Assuming a three-level decomposition, the lowpass and bandpass outputs can be written respectively as,

$$
\begin{aligned}
& \tilde{X}_{0, W T}(z)=\tilde{F}_{O, W T}(z) \tilde{X}(z) \downarrow 8 \\
& \tilde{X}_{i, W T}(z)=\tilde{F}_{i, W T}(z) \tilde{X}(z) \downarrow 2^{4-i}, i=1,2,3 \\
& \text { where } \quad \tilde{F}_{i, W T}(z)=\left\{\begin{array}{cc}
\tilde{H}(z) \tilde{H}\left(z^{2}\right) \tilde{H}\left(z^{4}\right) & i=0 \\
\tilde{G}(z) & i=3 \\
\tilde{G}\left(z^{2^{3-i}} \prod_{j=1}^{3-i} \tilde{H}\left(z^{j}\right)\right. & i=1,2
\end{array}\right.
\end{aligned}
$$

and $\tilde{H}(z)$ and $\tilde{G}(z)$ are lowpass and bandpass filter coefficients. Considering the specification of JPEG2000 (Part I and II) and the popularity in research usage, Haar, Daubechies-4 (DB4), Biorthogonal-(9,3) (B93) and Biorthogonal- $(9,7)$ kernels are selected to define them. 


\subsection{Comparison between BDCT and WT filters}

Owing to the passband difference between $\tilde{F}_{u, D C T}(z)$ and $\tilde{F}_{i, W T}(z)$, a few BDCT filters should be concatenated together to match WT filter. According to their magnitude spectra similarity in [4], the BDCT filters are combined as the following to compare with WT filters. They are,

$$
\begin{array}{lll}
\text { Lowpass: } & \tilde{F}_{0, W T}(z), \tilde{F}_{0, D C T}(z) \\
\text { bandpass 1: } & \tilde{F}_{1, W T}(z), \tilde{F}_{1, D C T}(z) \\
\text { bandpass 2: } & \tilde{F}_{2, W T}(z), \tilde{F}_{2, D C T}(z)+\tilde{F}_{4, D C T}(z) \\
\text { bandpass 3: } & \tilde{F}_{3, W T}(z), \tilde{F}_{4, D C T}(z)+\tilde{F}_{5, D C T}(z)+ \\
& & \tilde{F}_{6, D C T}(z)+\tilde{F}_{7, D C T}(z)
\end{array}
$$

\section{FEATURE EXTRACTION}

Even though BDCT and WT filters are very similar, compression may degrade their output similarity as JPEG and JPEG2000 employ different compression schemes such as quantization. In this section, we consider feature extraction directly from BDCT and WT coefficients with images compressed by JPEG and JPEG2000 to various degrees. The BDCT coefficients from all blocks $\tilde{X}_{m, n}(u, v)$ with the same values of $u$ and $v$ are concatenated to form a wavelet subband structure $B_{j}$, i.e.,

$$
B_{j} \in\left\{X_{m, n}\left(\varepsilon_{j}\right) \mid \varepsilon_{j} \in(u, v), u \in n_{u_{j}}, v \in n_{v_{j}}\right\}
$$

where $(m, n)$ represents the block location, $\varepsilon_{j}$ defines the coefficient coordinates at $j$ th subband, $n_{u_{j}}$ and $n_{v_{j}}$ are set of coordinate $(u, v)$ defined as in table 1. This concatenation is concluded from the common subband filtering model. $S B_{j}$ is used for an easier reference to the concatenated BDCT and WT subband. Image features can then be extracted from $S B_{j}$ for direct image retrieval.

\begin{tabular}{|c|c|c|c|c|c|}
\hline$j$ & $\begin{array}{c}\text { Subband } \\
\text { name }\end{array}$ & $\begin{array}{c}\text { Concatenated } \\
\text { BDCT subband }\end{array}$ & $\begin{array}{c}\text { Comparable WT } \\
\text { subband }\end{array}$ & $n_{u_{j}}$ & $n_{v_{j}}$ \\
\hline 0 & $S B_{0}$ & $B_{0}$ & $L L$ & 0 & 0 \\
\hline 1 & $S B_{1}$ & $B_{1}$ & $L H_{3}$ & 0 & 1 \\
\hline 2 & $S B_{2}$ & $B_{2}$ & $H L_{3}$ & 1 & 0 \\
\hline 3 & $S B_{3}$ & $B_{3}$ & $H H_{3}$ & 1 & 1 \\
\hline 4 & $S B_{4}$ & $B_{4}$ & $L H_{2}$ & 0,1 & 2,4 \\
\hline 5 & $S B_{5}$ & $B_{5}$ & $H L_{2}$ & 2,4 & 0,1 \\
\hline 6 & $S B_{6}$ & $B_{6}$ & $H H_{2}$ & 2,4 & 2,4 \\
\hline 7 & $S B_{7}$ & $B_{7}$ & $L H_{1}$ & $0,1,2,3$ & $4,5,6,7$ \\
\hline 8 & $S B_{8}$ & $B_{8}$ & $H L_{1}$ & $4,5,6,7$ & $0,1,2,3$ \\
\hline 9 & $S B_{9}$ & $B_{9}$ & $H H_{1}$ & $4,5,6,7$ & $4,5,6,7$ \\
\hline
\end{tabular}

Table 1: Rearrangement of BDCT coefficients to form wavelet subband structure.

\subsection{Feature extraction in JPEG and JPEG2000}

\subsubsection{Energy}

Energy conservation after transformation is an important property of BDCT and WT. Denoting the coefficient of the BDCT and WT at coordinate $(x, y)$ as $S B_{j}(x, y)$, the $j$ th subband energy $E_{j}$ is given by,

$$
E_{j}=\sum_{x, y} S B_{j}(x, y)^{2}
$$

Significant subband gives a large $E_{j}$ whereas the insignificant one gives a small value. $E_{j}$ thus indicates the importance of a subband. Dominant coefficients are mainly due to sharp edges but do not exist frequently in most images. Thus, very few high frequency components in $S B_{j}$ for $j=\{7,8,9\}$ give low $E_{j}$. In contrast, all subbands $S B_{j}$ for $j=\{0, \ldots, 6\}$ contain dominant coefficients and thus the feature vector regarding energy is formed as,

$$
f^{E}=\left\{E_{0}, E_{1}, \ldots, E_{6}\right\}
$$

\subsubsection{Significance map}

Significance map consisting of dominant coefficients can well represent an overall image shape [5]. Mathematically, the significance map at $(x, y)$ is defined as,

$$
M_{j}(x, y)= \begin{cases}1 & \text { if } S B_{j}(x, y)>T_{j} \\ 0 & \text { otherwise }\end{cases}
$$

where $T_{j}$ is a threshold at the $j$ th subband. Any coefficient larger than $T_{j}$ is considered to be significant and marked ' 1 '. Otherwise it is regarded as insignificant and marked ' 0 '. $T_{j}$ varies from one subbands to another. It is a value which allows a fixed percentage of coefficients considered to be significant in each subband. Experiment shows that $30 \%$ is sufficient for further feature extraction.

\subsubsection{Moments of significance map}

User may want to search for similar objects which are shifted, scaled or rotated. Normalized central moments provide statistical measures which are scaling, translation and rotation invariant $[3,6]$. It is defined as,

$$
\eta_{p q}^{j}=u_{p q}^{j} /\left(u_{00}^{j}\right)^{\gamma}
$$

where $p$ and $q$ define the order at the $j$ th subband and

$$
\begin{gathered}
u_{p q}^{j}=\sum_{y=1}^{M} \sum_{x=1}^{N}(x-\bar{x})^{p}(y-\bar{y})^{q} M_{j}(x, y) \\
\gamma=(p+q) / 2+1, \bar{x}_{j}=u_{10}^{j} / u_{00}^{j}, \bar{y}_{j}=u_{01}^{j} / u_{00}^{j}
\end{gathered}
$$

2nd and 3rd order normalized central moments are calculated from the significance map in each subband in order to find out its variance and skewness. Variance indicates the spread of significant coefficients with respect to the centroid, whereas skewness indicates the symmetry along multiple directions. These seven moment values form another feature vector, i.e.,

$$
f_{S B_{j}}^{M}=\left\{\eta_{20}^{j}, \eta_{11}^{j}, \eta_{02}^{j}, \eta_{30}^{j}, \eta_{21}^{j}, \eta_{12}^{j}, \eta_{03}^{j}\right\}
$$

Our studies find that the moment values at all decomposition levels are more or less the same due to the high correlation between the subbands across all the levels. Thus, moments are evaluated at $S B_{j}$ for $j=$ $\{0,1,2,3\}$ at the highest decomposition level only, i.e.,

$$
f^{M}=\left\{f_{S B_{0}}^{M}, f_{S B_{1}}^{M}, f_{S B_{2}}^{M}, f_{S B_{3}}^{M}\right\}
$$




\subsection{Feature vector}

$f^{E}$ and $f^{M}$ form our resultant feature vector $f$ as,

$$
f=\left\{f^{E}, f_{S B_{0}}^{M}, f_{S B_{1}}^{M}, f_{S B_{2}}^{M}, f_{S B_{3}}^{M}\right\}
$$

This 35-dimensional feature vector is translation and rotation invariant. It can represent image features at multiple scales and directions.

\subsection{Distance Measure}

To search for relevant images, Euclidean distance measure $D_{p, q}$ between the query image $q$ and the indexing image $p$ is performed to quantity their similarity [7], i.e.,

$$
D_{p, q}=\sum_{k=1}^{35}\left(f_{k}^{q}-f_{k}^{p}\right)^{2}
$$

where $f_{k}^{q}$ and $f_{k}^{p}$ is the $\mathrm{k}^{\text {th }}$ element in the feature vector of $q$ and $p$ respectively. A small $D_{p, q}$ indicates that $p$ and $q$ bear common characteristics.

\subsection{Simulation of the proposed retrieval system}

Precision $p_{q}$ and recall $r_{q}$ of the query image $q$ can be used to quantify the retrieval accuracy [3]. Let $n_{c}, n_{m}$ and $n_{f}$ be the numbers of correct, missed and false candidates respectively, $p_{q}$ and $r_{q}$ are defined respectively as,

$$
\begin{aligned}
& p_{q}=n_{c} /\left(n_{c}+n_{f}\right) \times 100 \%=n_{c} / M \times 100 \% \\
& r_{q}=n_{c} /\left(n_{c}+n_{m}\right) \times 100 \%=n_{c} / N_{q} \times 100 \%
\end{aligned}
$$

$p_{q}$ is measured at the first $M$ retrieved images, i.e., retrieval size is $M . \quad r_{q}$ measures the performance of each image class which consists $N_{q}$ images. It is desirable that both $p_{q}$ and $r_{q}$ approach to $100 \%$. Average precision $p_{a v}$ and average recall $r_{a v}$ can evaluate our overall system performance over $K$ number of query images , i.e.,

$$
p_{a v}=\frac{1}{K} \sum_{q=1}^{K} p_{q} \text { and } r_{a v}=\frac{1}{K} \sum_{q=1}^{K} r_{q}
$$

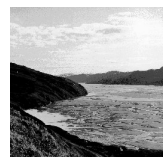

(a)

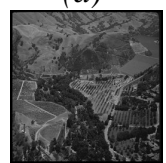

$(f)$



$(g)$
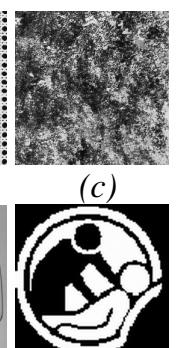

(h)

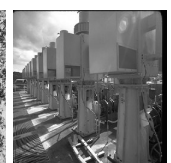

(d)

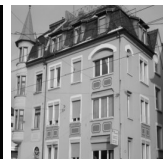

(e)
Fig. 1. Sample images from (a) natural scene, (b) structured texture pattern, (c) random texture pattern, (d) man-made object image, (e) building, (f) landscape, ( $g$ ) single object, (h) trade-mark and (i) human face.

\section{SIMULATION RESULTS}

Indexing performance at different compression ratios is investigated. Our database contains a total of 1800 images, which are equally cataloged into nine classes (Fig. 1), i.e., $N_{q}=200$. They are compressed with seven compression ratios ranging from 1.6:1 to $72: 1$. Using
JPEG images as the query image, the most similar JPEG2000 images compressed at the same compression ratio (CR) will be searched. Retrieval is classified as a correct case if both query and retrieved images come from the same catalogue; otherwise a false case.

\subsection{Discussion on precision}

To investigate the effect of compression and the use of wavelet kernel on image indexing, Fig. 2 plots the average precision of retrieving JPEG images from JPEG2000 images versus compression ratio when retrieval size is 1 . Curves are plotted separately for each compression kernel (i.e., Haar, DB4, B93 and B97 kernels) and each image kind (i.e., $K=200$ ). For Haar and DB4 wavelet kernels, the $p_{a v}$ of most image kinds are clustered at the range from $60 \%$ to $100 \%$ even under high CR and thus half of the relevant images can be retrieved. In contrast, curves for B93 and B97 kernels are widely distributed at the range from $20 \%$ to $99 \%$. This is because compression emphasizes the difference between BDCT and WT kernels [4]. The above analysis shows that the indexing accuracy of the first rank relevant image greatly depends on the CR and wavelet kernel.

Precision versus retrieval size in Fig. 3(a) tells how many relevant images are returned in the first $M$ retrieved images. Besides human face, $p_{a v}$ of all other image kinds drops about $10 \%$ to $30 \%$ when retrieval size increases. The declining of $p_{a v}$ is due to the loss of high frequency details in compression, which makes some image kinds indistinguishable. More than half of the image kinds have $p_{a v}$ of over $50 \%$. Hence, over half of relevant images can be retrieved by the proposed indexing algorithm.

To observe the effect of the kernel versus retrieval size, Fig. 3(b) plots the average precision for individual wavelet kernels. $p_{a v}$ of Haar, DB4, B97 and B93 are in descending order. Over half of the relevant data can be retrieved if JPEG2000 images are compressed by Part I standard, i.e., B97 kernel. Regarding the direct retrieval in multiple compressed domains, Haar kernel gives the best results.

\subsection{Discussion on recall}

To find out the proportion of relevant candidates to individual image kind, Fig. 4(a) plots the average recall of each image kind versus compression ratio. $r_{a v}$ is obtained by averaging all $r_{q}$ from each image kind (i.e. $K=200$ ) with the four compression kernels. In the first 200 ranks, about half of the images kinds miss less than half of their relevant images. In contrast, human face and single object images give an outstanding recall performance even under high CR due to their special image characteristics. $r_{a v}$ of most image kinds fluctuates when $\mathrm{CR}$ increases because the lost of fine details in compression cause those image kinds to be indistinguishable to each other. However, when CR is large enough, details are seriously lost and 
make them to be distinguishable again. Fig. 4(b) plots the average recall versus retrieval size. In general, $r_{a v}$ increases steadily as retrieval size increases. $r_{a v}$ of half of the image kinds goes up to $50 \%$ when retrieval size is 200 , which means half of related images can be retrieved.

In summary, our derived subband filtering model enables direct image indexing in JPEG and JPEG2000 domains without a full decompression. Despite of high compression ratio and the use of different wavelet kernel, a large number of relevant images can be retrieved.


Fig. 2: Average precision of each image kind versus compression ratio for different wavelet kernels.

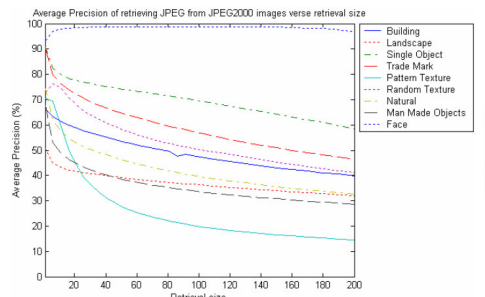

(a)

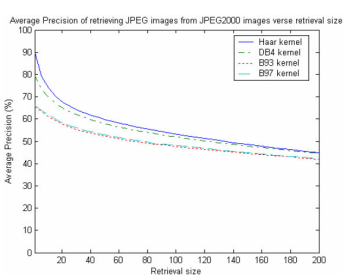

(b)
Fig. 3: Average precision versus retrieval size of (a) each image kind in ignorance of compressed kernel and compression ratio $(b)$ all image kinds with different compression kernels.

\section{CONCLUSION}

JPEG and JPEG2000 employ different transforms, namely the Block-based Discrete Cosine Transform (BDCT) and Wavelet Transform (WT) respectively. Subband filtering model shows that their filtering operations can be divided into four subbands and each indeed can be constructed by a common model, i.e., both consist of lowpass/bandpass filtering followed by a downsampling operation. Outputs from BDCT and WT have similar spectral characteristics even at high compression ratio irrespective of the choice of different wavelet kernels. Translation and rotation invariant features are extracted from the BDCT and WT subbands for image indexing. Precision and recall are used to quantify the retrieval accuracy of searching JPEG and JPEG2000 compressed images by our proposed algorithms. Although compression influences the retrieval accuracy, more than half of the relevant images can be retrieved even at high compression ratio.

Our throughout investigation concludes that relevant images can be searched from JPEG and JPEG2000 irrespective of the choice of kernel and the size of compression ratios. Both our theoretical and experimental studies prove that direct image indexing in different domains is possible without having a full decompression. Our derived subband filtering model can be applied to DCT and WT based compression schemes such as MPEG, MOTION JPEG and MOTION JPEG2000 for direct video retrieval in their respective domains.

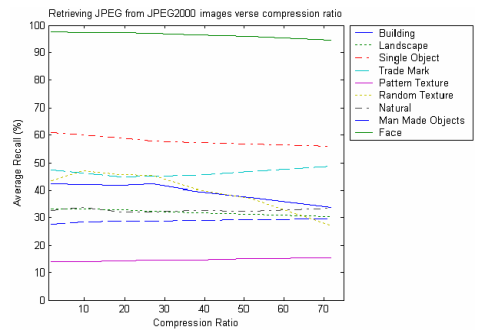

(a)

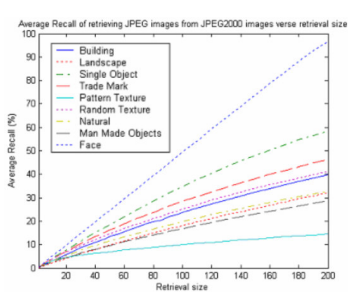

(b)
Fig. 4: Average recall versus (a) compression ratio and (b) retrieval size of each image kind.

\section{ACKNOWLEDGEMENTS}

This work is supported by the Centre for Multimedia Signal Processing (A-452), and the Internal Competitive Research Grants, The Hong Kong Polytechnic University (A-PD66). K.M. Au acknowledges the research studentships provided by the University.

\section{REFERENCES}

[1] M. Flickner, H. Sawhney, W. Niblack, J. Ashley, Q. Huang, B. Dom, M. Gorkani, J. Hafner, D. Lee, D. Petkovic, D. Steele and P. Yanker, "Query by Image and Video Content", IEEE Trans. Computer, Vol. 28, Issue 9, pp. 23-32, Sept. 1995.

[2] M. Shneier and M. Abdel-Mottaleb, "Exploiting the JPEG compression scheme for image retrieval," IEEE Trans.Pattern Anal. Machine Intell., Vol. 18, Issue 8, pp. 849-853, Aug. 1996

[3] K.C. Liang and C.C. Jay Kuo, "WaveGuide: a joint waveletbased image representation and description system," IEEE Trans.IP, Vol. 8, Issue 11, pp.1619-1629, Nov. 1999

[4] K.M. Au, N.F. Law and W.C. Siu, "Spatial-spectral feature analysis in JPEG and JPEG-2000", Int. Sym. Intelligent Multimedia, Video and Speech Processing," Vol.1, pp.378-381, Oct. 2004

[5] S.G Chang, Bin Yu and M. Vetterli, "Adaptive wavelet thresholding for image denoising and compression," IEEE Trans. IP, Vol. 9, Issue 9, pp. 1532-1546, Sept. 2000

[6] M. K. Hu, "Visual Pattern Recognition by Moment Invariants," IRE Transactions on Information Theory, pp.179187,1962

[7] D. Feng, W. C. Siu and H.J. Zhang, Multimedia Information Retrieval and Management: Technological Fundamentals and Applications, ISBN 3540002448, Springer, Chapter 1, 2003 